\title{
Article \\ Global Distributions of Clarireedia Species and Their In Vitro Sensitivity Profiles to Fungicides
}

\author{
Jian Hu ${ }^{1}$, Huangwei Zhang ${ }^{1}$, Yinglu Dong ${ }^{1}$, Shan Jiang ${ }^{1}$, Kurt Lamour ${ }^{2}$, Jun Liu ${ }^{1}$, Yu Chen ${ }^{1}$ and Zhimin Yang ${ }^{1, *}$ \\ 1 College of Agro-Grassland Science, Nanjing Agricultural University, Nanjing 210095, China; \\ jaffyhu@njau.edu.cn (J.H.); 2020220006@stu.njau.edu.cn (H.Z.); dongyinglu2020@163.com (Y.D.); \\ 2019120004@njau.edu.cn (S.J.); liujun825@njau.edu.cn (J.L.); cyu801027@njau.edu.cn (Y.C.) \\ 2 Department of Entomology and Plant Pathology, University of Tennessee, Knoxville, TN 37996, USA; \\ klamour@utk.edu \\ * Correspondence: nauyzm@njau.edu.cn
}

Citation: Hu, J.; Zhang, H.; Dong, Y.; Jiang, S.; Lamour, K.; Liu, J.; Chen, Y.; Yang, Z. Global Distributions of Clarireedia Species and Their In Vitro Sensitivity Profiles to Fungicides. Agronomy 2021, 11, 2036. https:// doi.org/10.3390/agronomy11102036

Academic Editors: Carlos García Delgado

Received: 31 August 2021

Accepted: 5 October 2021

Published: 11 October 2021

Publisher's Note: MDPI stays neutral with regard to jurisdictional claims in published maps and institutional affiliations.

Copyright: (c) 2021 by the authors. Licensee MDPI, Basel, Switzerland. This article is an open access article distributed under the terms and conditions of the Creative Commons Attribution (CC BY) license (https:// creativecommons.org/licenses/by/ $4.0 /)$.

\begin{abstract}
Dollar spot is reported to be caused by multiple Clarireedia species and is a serious problem on many turfgrasses around the world. To our knowledge, the distribution of different Clarireedia species and their sensitivity profiles to fungicides remains unknown. In this study, a total of 275 isolates were characterized by ITS sequence. Amounts of 124, 59 and 75 isolates were identified as C. jacksonii, C. monteithiana and C. paspali, respectively, while each species of C. homoeocarpa and C. bennettii had only five isolates. Four and three isolates were identified as two potential new species, which remained to be further characterized. C. jacksonii and C. monteithiana were distributed worldwide, while C. paspali was restricted to China. Of the isolates with host information, $81 \%$ (93/115) and 19\% (22/115) of C. jacksonii isolates were collected from C3 and C4 plants, respectively, $97 \%(56 / 58)$ of the C. monteithiana isolates were collected from C4 plants and all C. paspali isolates were collected from C4 plants. The coexistence of different Clarireedia species on the same C4 host type in the same locales was found in Shanghai (Paspalum vaginatum), Jiangsu (Paspalum vaginatum) and Florida (Cynodon dactylon). The study revealed that differential fungicide sensitivity patterns were observed in different species in Clarireedia for the first time. Similar differential sensitivity profiles were also found in the locales with coexistence of at least two species. The findings from this study suggest that the adjacent coexistence of different Clarireedia species and the differential fungicide sensitivity profiles of different species will complicate dollar spot disease control.
\end{abstract}

Keywords: turfgrass; Clarireedia; dollar spot; fungicide sensitivity; distribution

\section{Introduction}

Dollar spot, caused by the Clarireedia species (formerly Sclerotinia homoeocarpa), is one of the most economically important diseases of turfgrass worldwide [1,2]. A total of five distinct pathogenic species causing dollar spot within the genus Clarireedia have been documented [1,2], and it is speculated that additional species of Clarireedia may exist, possibly based on geographical distributions [2,3]. The five species in Clarireeida include the type species for the genus, C. homoeocarpa and four recently characterized species, $C$. bennettii, C. jacksonii, C. monteithiana and C. paspali.

Among the five species of Clarireedia that have been identified, $C$. homoeocarpa has only been found in the United Kingdom, whereas C. bennettii is primarily found in the United Kingdom and the United States [4]. The two species appear to be historical and represent a minority of the isolates which cause dollar spot. The modern species with worldwide distribution are C. jacksonii and C. monteithiana, which are also widely distributed in China [1]. C. paspali is an emerging species which frequently recovered from seashore paspalum (Paspalum vaginatum). It has not been reported in regions or countries other than eastern and southern China [1]. However, the collection of more isolates from diverse regions around the world is needed to understand if the C. paspali is geographically 
restricted. C. monteithiana and C. paspali isolates are predominantly collected from C4 turfgrass [1,2], suggesting a host preference in the two species. However, in contrast to a previous finding [2], C. jacksonii is not restricted to C3 turfgrass hosts and could be recovered from C4 turfgrass at a high frequency in China [1]. Different species of Clarireedia have been found on the same host type, indicating that they are host adapted; complicating dollar spot management. It is imperative to understand the role of geography and host type for the distributions of different Clarireedia species in order to most effectively manage dollar spot.

Management of dollar spot is the top priority in the turfgrass industry and requires high inputs of chemical fungicides. Multiple fungicides from four different chemical classes, including benzimidazole carbamate (MBC), dicarboximide, sterol demethylation inhibitor (DMI) and succinate dehydrogenase inhibitor (SDHI) fungicides, have been developed and marketed for dollar spot control [5]. However, repeated fungicidal applications select for isolates of Clarireedia species with reduced sensitivity to fungicides with differing modes of actions (MOAs) [5-11]. It is necessary to monitor the development of fungicide sensitivity in Clarireedia species, which is important for subsequent fungicide applications for dollar spot control. To our knowledge, all existing studies on fungicide sensitivity in Clarireedia species have considered the isolates as a single species (formerly S. homoeocarpa), and comparative analysis in fungicide sensitivity has mostly focused on isolates collected from different locales (e.g., golf courses) rather than those from different species $[5,12,13]$. Meanwhile, it has been demonstrated that there are morphological, vegetative, pathogenic and mating-type differences between C. jacksonii and C. monteithiana [4,14]. These findings warrant us an investigation into whether different Clarireedia species show differential sensitivity profiles to fungicides or different patterns in developing fungicide resistance.

The objectives of this study were to (i) identify dollar spot isolates into specific species based on a partial internal transcribed spacer (ITS) sequence, and characterize their global distributions, (ii) determine the sensitivity of different Clarireedia species in vitro to thiophanate-methyl (TM), iprodione, propiconazole and boscalid, and (iii) analyze if different Clarireedia species show differential sensitivity patterns to these fungicides.

\section{Materials and Methods}

\subsection{Medium and Fungicides}

Minimum medium (MM) consisted of $10 \mathrm{~g}$ glucose, $1.5 \mathrm{~g} \mathrm{~K}_{2} \mathrm{HPO}_{4}, 2 \mathrm{~g} \mathrm{KH}_{2} \mathrm{PO}_{4}, 1 \mathrm{~g}$ $\left(\mathrm{NH}_{4}\right)_{2} \mathrm{SO}_{4}, 0.5 \mathrm{~g} \mathrm{MgSO} .7 \mathrm{H}_{2} \mathrm{O}, 2 \mathrm{~g}$ yeast extract, and $12.5 \mathrm{~g}$ agar per liter of distilled water. Technical grade thiophanate-methyl (TM, 95\% active ingredient), iprodione (98\% active ingredient), propiconazole (96.5\% active ingredient), and boscalid (98.5\% active ingredient) were purchased from Zhejiang Heben Pesticide \& Chemicals Co., Ltd. (Wenzhou, China) and used for in vitro assays in this study. The fungicides were dissolved in acetone to provide stock solutions at $100 \mathrm{mg} \mathrm{mL}^{-1}$. Solutions were sealed with Parafilm, stored at $4{ }^{\circ} \mathrm{C}$ and diluted as required.

\subsection{Sample Collection and Pathogen Isolation}

Dollar spot samples were collected from 13 golf courses from May 2008 to September 2016. For dollar spot control, all golf courses were documented to have a history of benzimidazole and DMI fungicides applications, five golf courses (BA, BHA, BHU, LFT, LKS) had utilized dicarboximide fungicides, and three golf course (LFT, LKS, QHB) had utilized SDHI fungicides. Samples were collected from different host types, including Agrostis stolonifera, Poa pratensis, Cynodon dactylon, and Paspalum vaginatum (Table S1).

The Clarireedia species was isolated from leaf tissue with symptoms of dollar spot using the method described previously [6]. Identification of the Clarireedia species was based on cultural morphology. Five plugs ( $5 \mathrm{~mm}$ in diameter) of each isolate were put into a 2 -mL microtube, immersed by $20 \%$ glycerin and stored long-term at $10{ }^{\circ} \mathrm{C}$. 


\subsection{Sequencing of Internal Transcribed Spacer Regions}

Clarireedia species cultures were incubated on $\mathrm{MM}$ at $25^{\circ} \mathrm{C}$ for four days to produce enough mycelium for DNA extraction. Mycelial mats of each isolate were scraped from MM using a sterilized scalpel and transferred into a 2-mL microcentrifuge tube. Genomic DNA was extracted using the CTAB method described previously [15].

The internal transcribed spacer (ITS) region of ribosomal RNA gene proved useful to differentiate different species in the genus Clarireedia in previous studies [1,2,4], and the phylogenetic tree constructed from ITS produced a topology similar to the combined multi-locus dataset [1,2]. Therefore, we used the ITS sequence for species identification instead of multi-locus molecular markers. To molecularly characterize fungal isolates, the partial ITS sequence was amplified using the ITS4 and ITS5 primer pairs [16].

PCR amplifications were accomplished in $30 \mu \mathrm{L}$ reactions containing $1 \times$ TSINGKE master mix (Tsingke Biotech Co. Ltd., Nanjing, China), $200 \mathrm{nM}$ of each primer and $50 \mathrm{ng}$ of genomic DNA. The PCR cycling consisted of an initial preheating step at $94{ }^{\circ} \mathrm{C}$ for $2 \mathrm{~min}$, followed by 30 cycles of $94{ }^{\circ} \mathrm{C}$ for $15 \mathrm{~s}, 57^{\circ} \mathrm{C}$ for $30 \mathrm{~s}$ and $72{ }^{\circ} \mathrm{C}$ for $1 \mathrm{~min}$ and a final extension step at $72{ }^{\circ} \mathrm{C}$ for $10 \mathrm{~min}$. PCR products were detected by gel electrophoresis using a $1 \%$ agarose gel. The DNA fragment was excised from the agarose gel and purified using a TianGEN gel purification kit (DP209-03, TianGEN Biotech Co. Ltd., Beijing, China). The PCR products were sent to Sangon Biotech Co. Ltd. (Shanghai, China) for sequencing. Some ITS sequences (62 isolates) have been submitted to GenBank in a previous study [1], and more ITS sequences (51 isolates) were submitted in this study (Table S1).

\subsection{Species Identification}

The ITS fragments for all isolates were sequenced prior to assembly and then aligned using the ClustalW algorithm implemented in MEGA v7 [17]. ITS sequences of 162 Clarireedia isolates were also retrieved from GenBank for alignment and were used to construct a phylogenetic tree. Alignments were manually checked with characteristics weighted equally. Gaps were completely deleted. Maximum likelihood trees were generated using the Tamura 3-parameter model in MEGA v7 and tested with 1000 bootstrap replicates [17]. A 50\% majority-rule consensus tree was estimated. The aligned sequences were analyzed together with the ITS sequences of C. paspali (MH392087), C. homoeocarpa (MF964322), C. bennettii (MF964321), C. jacksonii (MF964320), C. monteithiana (KF545306) type strain obtained from GenBank. The ITS sequences of Rutstroemia firma (MH861930) and R. bolaris (LT158432) were included as outgroup members. Isolates grouped together with the type strains were defined to be the same species as the type strains. Otherwise, they were defined as Clarireedia sp.

\subsection{In Vitro Assessment of Fungicide Sensitivity}

A subset of isolates identified as different Clarireedia species were tested for fungicide sensitivity. Sensitivities of Clarireedia isolates to TM, iprodione, propiconazole, and boscalid were determined in vitro by using mycelia growth assays [5]. Agar plugs (5 $\mathrm{mm}$ in diameter) containing actively growing mycelium were transferred to $\mathrm{MM}$ and $\mathrm{MM}$ amended with propiconazole, iprodione and boscalid at $0.1,1$ and $10 \mu \mathrm{g} \mathrm{mL}^{-1}$, respectively. These concentrations have been reported to be effective discriminatory doses in our recent paper and other previous studies $[5,18,19]$. Each isolate was tested on three replicates of each fungicide concentration and the experiment was performed twice. Owing to the qualitative nature of Clarireedia isolates resistance to TM [19], a single discriminatory concentration of $10 \mu \mathrm{g} \mathrm{mL} \mathrm{m}^{-1}$ was used for in vitro sensitivity screening. Resistance to TM was scored $5 \mathrm{~d}$ after incubation at $20^{\circ} \mathrm{C}$ for the presence of mycelium growth on TM-amended MM $[18,19]$. Mean radial mycelial growth for propiconazole, iprodione and boscalid was scored $3 \mathrm{~d}$ after incubation at $25{ }^{\circ} \mathrm{C}$. Relative mycelia growth (RMG) value was calculated using the formula: $\% \mathrm{RMG}=($ mean fungicide amended $\mathrm{MM} /$ mean nonamended $\mathrm{MM}) \times 100$. RMG values for individual isolates from different species were compared and separated at $p<0.05$ according to Fisher's least significant difference test implemented in Data Process- 
ing System software (version 7.05, Hangzhou RuiFeng Information Technology Co. Ltd., Hangzhou, China)

\section{Results}

\subsection{Species Identification}

A total of 275 isolates were characterized by ITS sequences and all isolates fell into seven distinct groups. The three main groups included 124, 59 and 75 isolates which were identified as C. jacksonii, C. monteithiana and C. paspali, respectively. Two groups with five isolates in each were identified as $C$. homoeocarpa and $C$. bennettii. Four and three isolates in the remaining two groups were identified as the potential new species in Clarireedia (Clarireedia sp. 1 and Clarireedia sp. 2) (Table 1 and Table S1).

Table 1. The information of Clarireedia species identified by partial internal transcribed spacer (ITS) sequence in this study.

\begin{tabular}{|c|c|c|c|c|c|c|}
\hline \multirow{2}{*}{ Species } & \multirow{2}{*}{ No. of Isolates } & \multirow{2}{*}{ History } & \multicolumn{3}{|c|}{ Host Type } & \multirow{2}{*}{ Distribution $^{b}$} \\
\hline & & & C3 & $\mathrm{C} 4$ & N/A ${ }^{a}$ & \\
\hline Clarireedia homoeocarpa & 5 & 1937-2008 & 4 & 0 & 1 & United Kingdom \\
\hline Clarireedia bennettii & 5 & 1937-1973 & 0 & 0 & 5 & $\begin{array}{l}\text { The Netherlands, United Kingdom, USA } \\
\text { Canada, China (5), Ireland, Italy, Japan (4), }\end{array}$ \\
\hline Clarireedia jacksonii & 124 & 1972-2016 & 93 & 22 & 9 & $\begin{array}{c}\text { The Netherlands, Spain, United Kingdom, } \\
\text { USA (16) }\end{array}$ \\
\hline Clarireedia monteithiana & 59 & 2001-2016 & 2 & 56 & 1 & $\begin{array}{l}\text { Canada, China (5), Dominican Republic, } \\
\text { Japan (5), USA (7) }\end{array}$ \\
\hline Clarireedia paspali & 75 & 2012-2016 & 0 & 75 & 0 & China (3) \\
\hline Clarireedia sp. 1 & 4 & 2015 & 0 & 4 & 0 & China (1) \\
\hline Clarireedia sp. 2 & 3 & 2005-2013 & 3 & 0 & 0 & Japan (2), Norway \\
\hline Total & 275 & & 102 & 157 & 16 & - \\
\hline
\end{tabular}

a: N/A represents isolates with no known host information; ${ }^{b}$ : number of states or provinces in a country reported with Clarireedia species. was shown in the bracket.

\subsection{Global Distributions of Clarireedia Species}

C. homoeocarpa was found to be restricted to the United Kingdom from 1937 to 2008 , and C. bennettii could be found in the Netherlands, United Kingdom and the United States historically (from 1937 to 1973). C. jacksonii and C. monteithiana were found to be distributed worldwide (Figure 1 and Table 1), and they were first documented in 1972 and 2001 respectively. When taking USA and China as examples, C. monteithian was mainly distributed in the transitional zones and southern regions, while C. jacksonii could be found in both northern and southern regions (Figure 1). C. paspali was only found in eastern and southern China and was first reported in 2012 (Figure 1 and Table S1). One potential new species (Clarireedia sp. 1) was found in only one locale in Hainan, China. The other potential new species (Clarireedia sp. 2) had three isolates, which were collected from Kyoto and Shizuoka in Japan, and from Scandinavia in Norway (Table S1). 


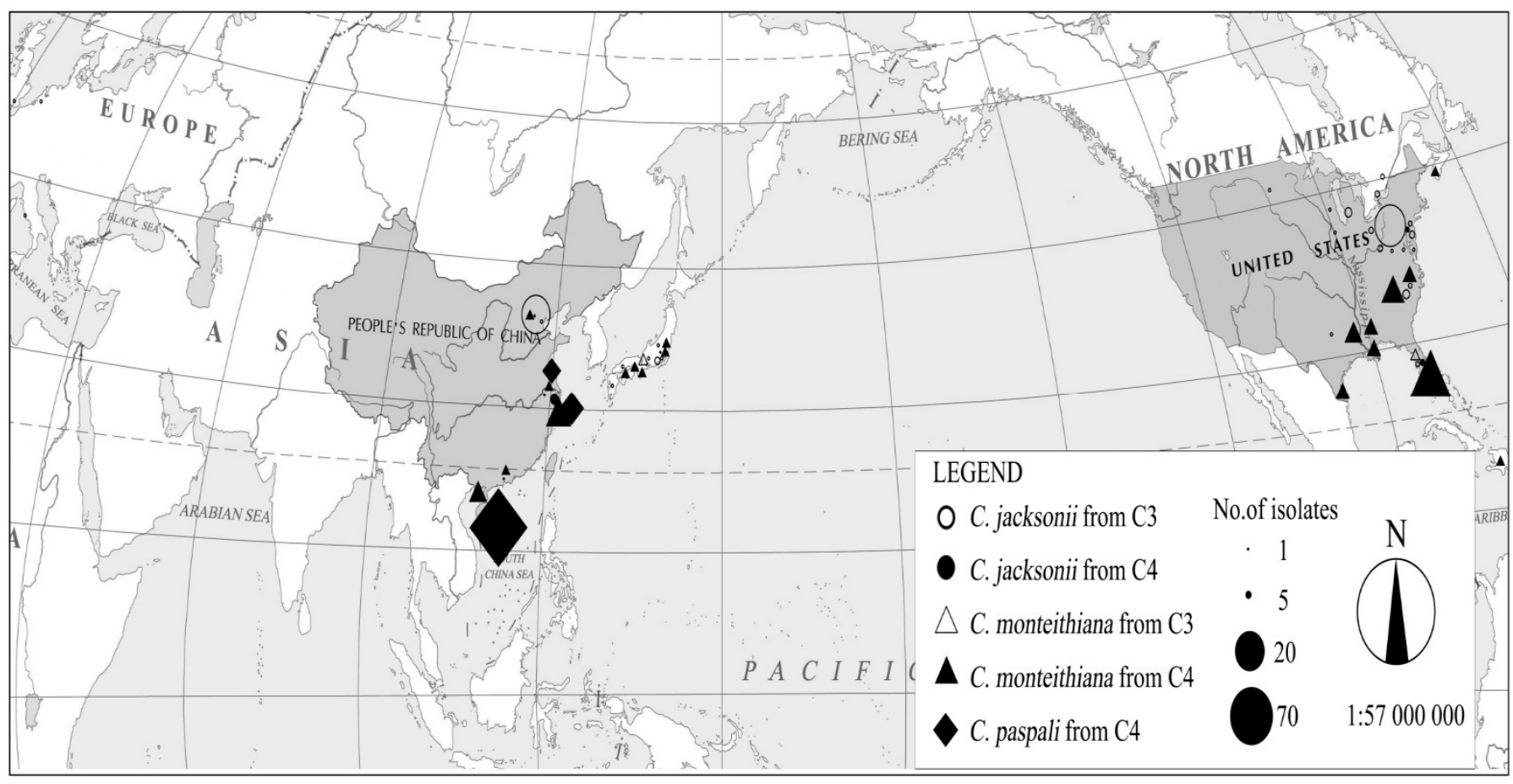

Figure 1. Global distributions of different Clarireedia species.

\subsection{Host Preference of Clarireedia Species}

Of the 115 C. jacksonii isolates with host information, 81\% (93/115) and 19\% (22/115) were collected from C3 and C4 plants, respectively (Table 1). A proportion of 97\% (56/58) of the C. monteithiana isolates were collected from C4 plants (Table 1). Two isolation events were found on C3 plants in Florida (Accession No. PFH0479) and Hyogo in Japan (Accession No. AB647342) (Table S1). One of the two events was found in the locale in Florida with C3 and C4 turfgrass growing adjacently [14]. All of the C. paspali isolates were collected from C4 plants (Table 1). Clarireedia sp. 1 and Clarireedia sp. 2 were solely recovered from C4 and C3 respectively (Table 1). The coexistence of different Clarireedia species on the same C4 host type in the same locales was found in Shanghai (Paspalum vaginatum), Jiangsu (Paspalum vaginatum) and Florida (Cynodon dactylon) (Table S1).

\subsection{In Vitro Sensitivity Profiles of Clarireedia Species}

Of the 88 Clarireedia species isolates that were tested, 24 were identified to be resistant to TM, 7 of the 24 TM-resistant isolates from six golf courses were C. paspali, and the remaining 17 TM-resistant isolates from nine golf courses were C. jacksonii. Of the 22 . jacksonii isolates, 17 were resistant to TM and 7 of the 56 C. paspali isolates were resistant to $\mathrm{TM}$, while none of the $10 \mathrm{C}$. monteithiana isolates from three golf courses were resistant to TM (Tables 2 and S2).

For propiconazole, the RMG of individual isolates ranged from 17.02 to $78.87,14.29$ to 39.71, and 4.29 to 45.00 for C. jacksonii, C. monteithiana and C. paspali, respectively. A significantly higher mean RMG was observed in C. jacksonii isolates against propiconazole $(p<0.001)$ (Tables 2 and S2).

For iprodione, the RMG of individual isolates ranged from 8.77 to $71.79,31.94$ to 44.12 , and 0.00 to 62.79 for C. jacksonii, C. monteithiana and C. paspali, respectively. No significant difference was observed in the mean RMG for different Clarireedia species against iprodione $(p>0.05)$ (Tables 2 and S2). 
Table 2. Sensitivity profiles of the three Clarireedia species to thiophanate-methyl, propiconazole, iprodione, and boscalid.

\begin{tabular}{|c|c|c|c|c|c|c|c|c|c|}
\hline \multirow{3}{*}{ Species } & \multirow{3}{*}{$\begin{array}{l}\text { No. of } \\
\text { Locations }\end{array}$} & \multirow{3}{*}{$\begin{array}{l}\text { No. of } \\
\text { Isolates }\end{array}$} & \multirow{3}{*}{$\begin{array}{c}\text { No. of Isolates } \\
\text { Resistant to } \\
\text { Thiophanate-Methyl }\end{array}$} & \multicolumn{6}{|c|}{ Relative Mycelial Growth (\%) } \\
\hline & & & & \multicolumn{2}{|c|}{ Propiconazole } & \multicolumn{2}{|c|}{ Iprodione } & \multicolumn{2}{|c|}{ Boscalid } \\
\hline & & & & Range & Mean ${ }^{a}$ & Range & Mean & Range & Mean \\
\hline Clarireedia jacksonii & 9 & 22 & 17 & $17.02-78.87$ & $48.67 \mathrm{a}$ & $8.77-71.79$ & $34.67 \mathrm{a}$ & $23.46-67.57$ & $42.85 \mathrm{a}$ \\
\hline $\begin{array}{c}\text { Clarireedia } \\
\text { monteithiana }\end{array}$ & 3 & 10 & 0 & $14.29-39.71$ & $28.32 \mathrm{~b}$ & $31.94-44.12$ & $36.97 \mathrm{a}$ & $35.71-60.87$ & $46.51 \mathrm{a}$ \\
\hline Clarireedia paspali & 6 & 56 & 7 & $4.29-45.00$ & $27.75 \mathrm{~b}$ & $0.00-62.79$ & $34.11 \mathrm{a}$ & $0.00-23.73$ & $14.01 \mathrm{~b}$ \\
\hline
\end{tabular}

a Means followed by different letters in the same column are significantly different according to Fisher's least significant difference test at $p=0.05$.

For boscalid, the RMG of individual isolates ranged from 23.46 to $67.57,35.71$ to 60.87 , and 0.00 to 23.73 for C. jacksonii, C. monteithiana and C. paspali, respectively. A significantly lower mean RMG for $C$. paspali against boscalid was observed $(p<0.001)$ (Tables 2 and S2).

\subsection{In Vitro Sensitivity Profiles at Locales with Clarireedia Species Coexistence}

In the four golf courses with Clarireedia species co-existing, similar in vitro sensitivity profiles to the four fungicides were observed (Table 3). TM-resistance was found in $C$. jacksonii and C. paspali isolates, but not in C. monteithiana isolates. The RMG of C. jacksonii isolates to propiconazole was higher than that of C. paspali and/or C. monteithiana isolates at each golf course. No apparent differentiation in RMG for iprodione was observed among different species in the same locales. The RMG for boscalid was lower in all C. paspali isolates when compared to that of the $C$. jacksonii or $C$. monteithiana isolates co-existing in the same locales (Table 3).

Table 3. Fungicide sensitivity in Clarireedia isolates from Paspalum vaginatum in the four locales with at least two species coexistence.

\begin{tabular}{|c|c|c|c|c|c|c|c|c|c|}
\hline \multirow{2}{*}{ Code } & \multirow{2}{*}{ Species $^{\text {a }}$} & \multirow{2}{*}{$\mathrm{TM}_{\text {Sensitivity }}{ }^{\mathrm{b}}$} & \multicolumn{3}{|c|}{ Relative Mycelium Growth (\%) ${ }^{b}$} & \multirow{2}{*}{ Collection Year } & \multirow{2}{*}{ Site } & \multirow{2}{*}{ Location } & \multirow{2}{*}{ Province } \\
\hline & & & $\mathbf{P}$ & $\mathbf{Y}$ & D & & & & \\
\hline BH15-5 & $\mathrm{CM}$ & $\mathrm{S}$ & 14.29 & 36.11 & 40.82 & September 2016 & Fairway & Binhai Golf Club & Shanghai \\
\hline BH17-8 & $\mathrm{CM}$ & $S$ & 30.43 & 36.76 & 60.87 & September 2016 & Fairway & Binhai Golf Club & Shanghai \\
\hline BH18-2 & $\mathrm{CM}$ & $S$ & 34.38 & 34.68 & 59.38 & September 2016 & Fairway & Binhai Golf Club & Shanghai \\
\hline BH18-4 & $\mathrm{CM}$ & $S$ & 39.66 & 31.94 & 42.11 & September 2016 & Fairway & Binhai Golf Club & Shanghai \\
\hline BH18-4 & $\mathrm{CM}$ & $\mathrm{S}$ & 39.71 & 37.14 & 51.47 & September 2016 & Fairway & Binhai Golf Club & Shanghai \\
\hline BH6-4 & $\mathrm{CM}$ & $S$ & 20.59 & 38.24 & 42.65 & September 2016 & Fairway & Binhai Golf Club & Shanghai \\
\hline BH17-6 & $\mathrm{CJ}$ & $\mathrm{R}$ & 78.87 & 52.17 & 33.80 & September 2016 & Fairway & Binhai Golf Club & Shanghai \\
\hline LKS1-1 & $\mathrm{CJ}$ & $\mathrm{R}$ & 73.56 & 17.81 & 36.49 & September 2016 & Fairway & Links Golf Club & Shanghai \\
\hline LKS11-2 & $\mathrm{CJ}$ & $\mathrm{R}$ & 67.05 & 16.95 & 31.17 & September 2016 & Fairway & Links Golf Club & Shanghai \\
\hline LKS1-2 & CJ & $\mathrm{R}$ & 77.38 & 58.73 & 28.38 & September 2016 & Fairway & Links Golf Club & Shanghai \\
\hline LKS13-6 & CJ & $\mathrm{R}$ & 73.97 & 22.58 & 49.33 & September 2016 & Fairway & Links Golf Club & Shanghai \\
\hline LKS11-3 & $\mathrm{CP}$ & $\mathrm{R}$ & 21.21 & 27.45 & 18.57 & September 2016 & Fairway & Links Golf Club & Shanghai \\
\hline TC5-6 & $\mathrm{CJ}$ & $\mathrm{R}$ & 51.72 & 71.79 & 42.53 & September 2016 & Fairway & Xingdonghai Golf Club & Jiangsu \\
\hline TC8-3 & $\mathrm{CP}$ & $S$ & 10.71 & 36.21 & 20.43 & September 2016 & Fairway & Xingdonghai Golf Club & Jiangsu \\
\hline TC8-5 & $\mathrm{CP}$ & $S$ & 5.26 & 45.07 & 15.79 & September 2016 & Fairway & Xingdonghai Golf Club & Jiangsu \\
\hline TC12-4 & $\mathrm{CP}$ & $\mathrm{R}$ & 4.29 & 24.39 & 5.06 & September 2016 & Fairway & Xingdonghai Golf Club & Jiangsu \\
\hline TC10-4 & $\mathrm{CM}$ & $\mathrm{S}$ & 30.43 & 44.12 & 40.58 & September 2016 & Fairway & Xingdonghai Golf Club & Jiangsu \\
\hline TC5-8 & $\mathrm{CM}$ & $\mathrm{S}$ & 22.08 & 32.35 & 41.56 & September 2016 & Fairway & Xingdonghai Golf Club & Jiangsu \\
\hline TC8-6 & $\mathrm{CM}$ & $\mathrm{S}$ & 27.14 & 35.48 & 35.71 & September 2016 & Fairway & Xingdonghai Golf Club & Jiangsu \\
\hline XTH18F-4 & $\mathrm{CP}$ & $\mathrm{R}$ & 19.12 & 18.92 & 20.59 & September 2016 & Fairway & Xingtianhong Golf Club & Shanghai \\
\hline ХTH18T-5 & $\mathrm{CP}$ & $\mathrm{R}$ & 16.28 & 18.00 & 10.00 & September 2016 & Fairway & Xingtianhong Golf Club & Shanghai \\
\hline XTHA10-5 & $\mathrm{CP}$ & $\mathrm{R}$ & 23.29 & 14.52 & 5.56 & September 2016 & Fairway & Xingtianhong Golf Club & Shanghai \\
\hline ХTHA15T-7 & $\mathrm{CP}$ & $\mathrm{R}$ & 19.35 & 23.88 & 16.49 & September 2016 & Fairway & Xingtianhong Golf Club & Shanghai \\
\hline XTH27G-2 & $\mathrm{CJ}$ & $\mathrm{R}$ & 60.34 & 48.65 & 37.50 & September 2016 & Fairway & Xingtianhong Golf Club & Shanghai \\
\hline XTH27G-3 & $\mathrm{CJ}$ & $\mathrm{R}$ & 56.45 & 37.84 & 23.46 & September 2016 & Fairway & Xingtianhong Golf Club & Shanghai \\
\hline XTH27G-3 & $\mathrm{CJ}$ & $\mathrm{R}$ & 59.74 & 47.14 & 29.31 & September 2016 & Fairway & Xingtianhong Golf Club & Shanghai \\
\hline XTH27G-5 & CJ & $\mathrm{R}$ & 47.27 & 22.22 & 30.91 & September 2016 & Fairway & Xingtianhong Golf Club & Shanghai \\
\hline
\end{tabular}

${ }^{\mathrm{a}} \mathrm{CM}=$ Clarireedia monteithiana, $\mathrm{CJ}=$ Clarireedia jacksonii, $\mathrm{CP}=$ Clarireedia paspali. ${ }^{\mathrm{b}} \mathrm{TM}=$ Thiophanate-methyl, $\mathrm{S}=$ Sensitive, $\mathrm{R}=$ Resistant. ${ }^{\mathrm{c}}$ Relative mycelial growth of Clarireedia isolates for propiconazole, iprodione and boscalid were estimated on the discriminatory concentrations of $0.1,1$ and $10 \mu \mathrm{g} / \mathrm{mL}$ respectively. $\mathrm{P}=$ Propiconazole, $\mathrm{I}=$ Iprodione, and $\mathrm{B}=$ Boscalid.

\section{Discussion}

This study reported on the distribution of Clarireedia species around the world, and the differential sensitivity patterns of C. jacksonii, C. monteithiana, and C. paspali populations to TM, propiconazole and boscalid, but not to iprodione. The information presented here 
will have important implications for the management of dollar spot disease and future monitoring for the development of fungicide resistance in dollar spot pathogens.

In this study, $97 \%$ of the C. monteithiana isolates, all C. homoeocarpa, C. paspali and two potential new species isolates were exclusively collected from either C3 or C4 turfgrass, strongly indicating a host preference. Meanwhile, all isolates in Europe and the northern regions of the USA and China were $C$. jacksonii, which also indicates a strong geographic distribution (Figure 1). We propose that both host type and geography are likely to influence the distribution of Clarireedia species. Previous studies also revealed that $C$. jacksonii isolates were different from C. monteithiana in many aspects, including pathogenicity and mycelial melanization processes $[4,14]$. Different pathogenicity may cause host preference, while melanization may help isolates resist environmental stress factors present at different geographic locations [14]. It is possible that different Clarireedia species have undergone some degree of host specialization, evolving different host specific virulence factors [20]. When spread to multiple regions around the world, some Clarireedia species (e.g., C. jacksonii) could be better adapted to the cold climate while others preferred warm climates [21] Further comparative genomics and competition studies among different Clarireedia species will aid in understand if they are selected by different host types or environmental factors.

It is still not clear how Clarireedia species have spread worldwide. Long distance dissemination by spores seems unlikely for Clarireedia species, because spore production was only rarely reported in C. homoeocarpa collected from fescues (Festuca sp.) in the United Kingdom [22]. Seeds or vegetative materials of turfgrass are the possible vectors for the long distance dissemination, which has been speculated in relevant studies [21,22]. In this study, isolates of the Clarireedia species from China were incorporated with world isolates for the analysis. Similarly, C. jacksonii and C. monteithiana were widely distributed in China, moreover, a new species (C. paspali) was also found to be distributed widely in eastern and southern China. It should be noted that turfgrass seeds or vegetative materials are mainly imported in China, which provides an optimal way for the long distance dissemination of Clarireedia species. One potential new species, found in Japan and Norway, further supports the possibility of human-mediated dissemination over long distance via seeds or vegetative materials of turfgrass. It is not known how the new species $C$. paspali emerged in China. It may have evolved through a recombination event in China, or may have been spread from other regions around the world via human-mediated dissemination, although it has not been reported in the places other than China. However, some unknown species have been reported at many regions around the world [2,21], suggesting the possibility of other origins of C. paspali. Further study, including on isolates of unknown species from worldwide collections, will aid in better understanding of how C. paspali emerged in China.

Differential sensitivities in Clarireedia species populations against multiple fungicides have been frequently reported at locations worldwide $[6,8,12,13,18,23]$. However, none of these studies reported if differential sensitivity patterns existed among different Clarireedia species. There are two main reasons which may explain why no such studies have been reported. Firstly, dollar spot was not characterized as being caused by different species until 2018. Formerly, it was reported to be caused by the fungus Sclerotinia homoeocarpa [2]; Secondly, most studies on fungicide sensitivity in Clarireedia species populations were restricted in certain regions or by the same host type, where only one species may exist. However, two or more Clarireedia species co-existed on the same host type at multiple locales in China and the United States, indicating that it is necessary to investigate the fungicide sensitivity patterns among different species in Clarireedia in these places.

No C. monteithiana isolates were resistant to TM, which may indicate that it is difficult to develop TM-resistance in C. monteithiana populations. A similar phenomenon was also indicated in a previous study, in which none of the F-type isolates were found to be resistant to benzimidazole fungicides [14]. F-type isolates were confirmed to be C. monteithiana in our study (Figure S1). Similar sensitivity patterns among different species were also observed in the fungicide propiconazole, the least mean RMG of C. monteithiana, suggesting that $C$. monteithiana may have difficulty developing resistance to propiconazole compared 
to C. jacksonii or C. paspali. Unlike TM and propiconazole, sensitivity of different Clarireedia species to boscalid seems inherently different, because almost all C. paspali isolates showed less RMG than that of C. jacksonii and C. monteithiana isolates, and most golf courses had no known history of SDHI fungicide application. The differential sensitivity patterns among the species observed in this study was further indicated by analyzing the locales with at least two Clarireedia species from the same turfgrass host. However, further studies are still needed to better understand the sensitivity patterns observed in this study, by collecting larger samples representing different species of Clarireedia from the same host in the same locales.

Difficulty in developing fungicide resistance has been reported in various studies [24-29]. Banno et al. identified isolates of B. cinerea from Japan that had two distinct $c y t b$ profiles; the first had a group I intron immediately after position 143, while the second one did not possess the intron. Isolates without the intron developed the G143A substitution and, consequently, were found to be highly resistant to the QoI fungicides; while isolates with the intron did not develop a high resistance to QoI fungicides [24]. An intron adjacent to position 143 in $c y t b$ has also been observed in other plant-pathogenic fungi, including Alternaria solani, Monilinia fructicola, Phyllosticta ampelicida, Fusarium effusum, Bipolaris oryzae, Guignardia citricarpa and Magnaporthe poae [25-30], and none of these showed a high resistance to QoI fungicides. Preliminary analysis showed a high frequency of introns gain or loss in the genomes of different Clarireedia species (unpublished data), suggesting insertions and deletions may play an important role in the differential sensitivity patterns of Clarireedia species. Further studies are needed to compare the targeted genes of TM and propiconazole among different Clarireedia species and investigate the role of insertions and deletions in key regions for fungicide sensitivity.

The inherent differential sensitivity among different species in the same genus or different isolates within the same species has also been reported [31-34]. Two possible mechanisms might be responsible for the differential sensitivity. Ueyama et al. reported that isolates of Rhizoctonia AG 4 are able to metabolize pencycuron and are less sensitive, whereas AG3 isolates cannot metabolize this fungicide and are sensitive [35]. Amino acid variations in the targeted genes of fungicides were reported as the other possible mechanism which may cause the differential sensitivity in Colletotrichum spp. and $B$. cinerea [31,33]. Wild-type isolates of B. cinerea in Florida [36], Germany [37], and China were frequently characterized as highly sensitive (HS) to boscalid, compared to other sensitive isolates, and amino acid polymorphisms in $S d h C$ are responsible for the HS isolates. By genetic transformation analyses, Shao et al. revealed that four different types of mutations in $S d h C$ could cause increased the sensitivity of wild-type $B$. cinerea isolates to boscalid and fluopyram, which included R-G1 (G85A, I93V, M158V and V168I), R-G2 (G37S), R-G3(I93V), and R-G4 (I79V, G85A and L151I) [33]. Phylogeny of genes for species identification and the potential targeted genes $(\mathrm{S} d h \mathrm{~B}, \mathrm{~S} d h \mathrm{C}$ and $\mathrm{S} d h \mathrm{D})$ of boscalid, as well as metabolism analysis, may be useful for better understanding if the differential boscalid sensitivity observed in Clarireedia species is inherent.

\section{Conclusions}

This study revealed the global distribution of different Clarireedia species. Both host type and geography are likely to influence the distribution of Clarireedia species. The coexistence of different Clarireedia species on the same C4 host type in the same locales was found in different regions. No C. monteithiana isolates were resistant to thiophanate-methyl or propiconazole. Differential sensitivity among different species was observed in terms of propiconazole and boscalid $(p<0.0001)$, but not in iprodione. The study will have important implications for dollar spot management, especially in the regions with the coexistence of different Clarireedia species.

Supplementary Materials: The following are available online at https:/ /www.mdpi.com/article/10 .3390/agronomy11102036/s1, Figure S1: Phylogenetic tree from maximum likelyhood analysis of the internal transcribed spacer sequences from global Clarireedia isolates. Numbers at each branch 
indicate percentage of occurrences of that branch in 1000 bootstrap replications. Isolates grouped together with the type strains were defined to be the same species as the type strains. Otherwise, they were defined as Clarireedia sp.; Table S1: Summary information of 275 global Clarireedia isolates.; Table S2: In vitro fungicide sensitivity profiles of the three Clarireedia species from different regions.

Author Contributions: Conceptualization, J.H.; methodology, H.Z.; software, H.Z. and Y.D.; validation, H.Z., Y.D. and S.J.; formal analysis, J.L.; investigation, J.H. and Z.Y.; resources, J.H.; data curation, J.H. and H.Z.; writing-original draft preparation, J.H. and H.Z.; writing-review and editing, J.H., Y.C. and K.L.; supervision, J.H.; project administration, J.H.; funding acquisition, J.H. All authors have read and agreed to the published version of the manuscript.

Funding: Natural Science Foundation of China, Grant Number: 31701810; Projects of Science and Technology in Northern Jiangsu, Grant Number: SZ-SQ20190009, SZ-SQ202068.

Institutional Review Board Statement: Not applicable.

Informed Consent Statement: Not applicable.

Data Availability Statement: Exclude this statement.

Acknowledgments: We thank the National Natural Science Foundation of China (31701810), and Projects of Science and Technology in Northern Jiangsu (SZ-SQ20190009 and SZ-SQ202068) for financial support of this research. We also thank China Scholarship Council for supporting Jian $\mathrm{Hu}$ as a visiting scholar at Michigan State University for one year.

Conflicts of Interest: The authors declare that none of the authors has competing interests.

\section{References}

1. Hu, J.; Zhou, Y.; Geng, J.; Dai, Y.; Ren, H.; Lamour, K. A new dollar spot disease of turfgrass caused by Clarireedia paspali. Mycol. Prog. 2019, 18, 1423-1435. [CrossRef]

2. Salgado-Salazar, C.; Beirn, L.A.; Ismaiel, A.; Boehm, M.J.; Carbone, I.; Putman, A.I.; Tredway, L.P.; Clarke, B.B.; Crouch, J.A. Clarireedia: A new fungal genus comprising four pathogenic species responsible for dollar spot disease of turfgrass. Fungal Biol. 2018, 122, 761-773. [CrossRef] [PubMed]

3. Espevig, T.; Brurberg, M.B.; Kvalbein, A. First report of dollar spot, Caused by Sclerotinia homoeocarpa, of creeping bentgrass in Norway. Plant Dis. 2015, 99, 287. [CrossRef] [PubMed]

4. Aynardi, B.A.; Jiménez-Gasco, M.M.; Uddin, W. Effects of isolates of Clarireedia jacksonii and Clarireedia monteithiana on severity of dollar spot in turfgrasses by host type. Eur. J. Plant Pathol. 2019, 155, 817-829. [CrossRef]

5. Stephens, C.M.; Kaminski, J. In vitro fungicide-insensitive profiles of Sclerotinia homoeocarpa populations from Pennsylvania and the surrounding region. Plant Dis. 2019, 103, 214-222. [CrossRef]

6. Hu, J.; Yang, J.Y.; Li, J.; Ma, Z.Y.; Yao, W.J.; Ren, H.Y.; Zhang, F.G.; Yang, G.W.; Sun, X.; Xiao, Y. Sensitivity of Sclerotinia homoeocarpa from turfgrass to thiophanate-methl, iprodione and propiconazole. Chin. J. Pestic. Sci. 2017, 19, 694-700.

7. Ostrander, J.C.; Todd, R.B.; Kennelly, M.M. Resistance of Kansas Sclerotinia homoeocarpa isolates to thiophanate-methyl and determination of associated $\beta$-tubulin mutation. Plant Health Prog. 2014, 15, 23-27. [CrossRef]

8. Popko, J.T., Jr.; Sang, H.; Lee, J.; Yamada, T.; Hoshino, Y.; Jung, G. Resistance of Sclerotinia homoeocarpa field isolates to succinate dehydrogenase inhibitor fungicides. Plant Dis. 2018, 102, 2625-2631. [CrossRef]

9. Sang, H.; Hulvey, J.; Popko, J.T., Jr.; Lopes, J.; Swaminathan, A.; Chang, T.; Jung, G. A pleiotropic drug resistance transporter is involved in reduced sensitivity to multiple fungicide classes in Sclerotinia homoeocarpa (F.T. Bennett). Mol. Plant Pathol. 2015, 16, 251-261. [CrossRef]

10. Sang, H.; Popko, J.T., Jr.; Chang, T.; Jung, G. Molecular mechanisms involved in qualitative and quantitative resistance to the dicarboximide fungicide iprodione in Sclerotinia homoeocarpa field isolates. Phytopathology 2017, 107, 198-207. [CrossRef]

11. Sang, H.; Hulvey, J.P.; Green, R.; Xu, H.; Im, J.; Chang, T.; Jung, G. A xenobiotic detoxification pathway through transcriptional regulation in filamentous fungi. mBio 2018, 9, e00457-18. [CrossRef]

12. Lee, J.W.; Choi, J.; Kim, J.W. Triazole fungicides sensitivity of Sclerotinia homoeocarpa in Korean golf courses. Plant Pathol. J. 2017, 33, 589-596. [CrossRef] [PubMed]

13. Putman, A.I.; Jung, G.; Kaminski, J.E. Geographic distribution of fungicide-insensitive Sclerotinia homoeocarpa isolates from golf courses in the northeastern United States. Plant Dis. 2010, 94, 186-195. [CrossRef] [PubMed]

14. Liberti, D.; Rollins, J.A.; Harmon, P.F. Evidence for morphological, vegetative, genetic, and mating-type diversity in Sclerotinia homoeocarpa. Phytopathology 2012, 102, 506-518. [CrossRef]

15. Chen, F.; Everhart, S.E.; Bryson, P.K.; Luo, C.; Song, X.; Liu, X.; Schnabel, G. Fungicide-induced transposon movement in Monilinia fructicola. Fungal Genet. Biol. 2015, 85, 38-44. [CrossRef]

16. White, T.J.; Bruns, T.; Lee, S.; Taylor, J. Amplification and direct sequencing of fungal ribosomal RNA genes for phylogenetics. In PCR Protocols: A Guide to Methods and Applications; Academic Press: Cambridge, MA, USA, 1990; Volume 18, pp. 315-322. 
17. Kumar, S.; Stecher, G.; Tamura, K. MEGA7: Molecular evolutionary genetics analysis version 7.0 for bigger datasets. Mol. Biol. Evol. 2016, 33, 1870-1874. [CrossRef] [PubMed]

18. Hu, J.; Deng, S.; Gao, T.; Lamour, K.; Liu, X.; Ren, H. Thiophanate-methyl resistance in Sclerotinia homoeocarpa from golf courses in China. Pestic. Biochem. Phys. 2018, 152, 84-89. [CrossRef] [PubMed]

19. Zhang, H.W.; Jiang, S.; Zhao, Z.Y.; Guan, J.; Dong, Y.L.; Hu, J.; Lamour, K.; Yin, S.X.; Yang, Z.M. Fungicide sensitivity of Clarireedia spp. isolates from golf courses in China. Crop Prot. 2021, 149, 105785. [CrossRef]

20. Chen, J.Y.; Liu, C.; Gui, Y.J.; Si, K.W.; Zhang, D.D.; Wang, J.; Short, D.P.G.; Huang, J.Q.; Li, N.Y.; Liang, Y.; et al. Comparative genomics reveals cotton-specific virulence factors in flexible genomic regions in Verticillium dahliae and evidence of horizontal gene transfer from Fusarium. New Phytol. 2018, 217, 756-770. [CrossRef]

21. Putman, A.I. Worldwide Population Biology of Sclerotinia homoeocarpa From Common Turfgrass Hosts and Mating Systems of the Pathogen and Closely Related Fungi. Ph.D. Thesis, North Carolina State University, Raleigh, NC, USA, 2013.

22. Rioux, R.A.; Shultz, J.; Garcia, M.; Willis, D.K.; Casler, M.; Bonos, S.; Smith, D.; Kerns, J. Sclerotinia homoeocarpa overwinters in turfgrass and is present in commercial seed. PLoS ONE 2014, 9, e110897. [CrossRef] [PubMed]

23. Koch, P.L.; Grau, C.R.; Jo, Y.K.; Jung, G. Thiophanate-methyl and propiconazole sensitivity in Sclerotinia homoeocarpa populations from golf courses in Wisconsin and Massachusetts. Plant Dis. 2009, 93, 100-105. [CrossRef]

24. Banno, S.; Yamashita, K.; Fukumori, F.; Okada, K.; Uekusa, H.; Takagaki, M.; Kimura, M.; Fujimura, M. Characterization of QoI resistance in Botrytis cinerea and identification of two types of mitochondrial cytochrome b gene. Plant Pathol. 2009, 58, 120-129. [CrossRef]

25. Chen, S.N.; Luo, C.X.; Hu, M.J.; Schnabel, G. Sensitivity of Colletotrichum Species, including C. fioriniae and C. nymphaeae, from peach to demethylation inhibitor fungicides. Plant Dis. 2016, 100, 2434-2441. [CrossRef]

26. Hu, J.; Yang, J.Y.; Li, J.; Yang, G.W.; Ren, H.Y. Azoxystrobin sensitivity in Magnaporthe poae populations collected from turfgrass in Beijing and analysis of its cytochrome b (Cytb) gene sequence. Chin. J. Pestic. Sci. 2018, 20, 33-40.

27. Kreis, R.A.; Dillard, H.R.; Smart, C.D. Population diversity and sensitivity to azoxystrobin of Alternaria brassicicola in New York State. Plant Dis. 2016, 100, 2422-2426. [CrossRef] [PubMed]

28. Pereira, W.V.; Primiano, I.V.; Morales, R.G.F.; Peres, N.A.; Amorim, L.; May De Mio, L.L. Reduced sensitivity to azoxystrobin of Monilinia fructicola isolates from Brazilian stone fruits is not associated with previously described mutations in the cytochrome $b$ gene. Plant Dis. 2017, 101, 766-773. [CrossRef]

29. Standish, J.R.; Avenot, H.F.; Brenneman, T.B.; Stevenson, K.L. Location of an intron in the cytochrome b gene indicates reduced risk of QoI fungicide resistance in Fusicladium effusum. Plant Dis. 2016, 100, 2294-2298. [CrossRef]

30. Wong, F.P.; Midland, S.L.; de la Cerda, K.A. Occurrence and distribution of QoI-resistant isolates of Colletotrichum cereale from annual bluegrass in California. Plant Dis. 2007, 91, 1536-1546. [CrossRef]

31. Chen, S.; Wang, Y.; Schnabel, G.; Peng, C.A.; Lagishetty, S.; Smith, K.; Luo, C.; Yuan, H. Inherent resistance to 14 alphademethylation inhibitor fungicides in Colletotrichum truncatum is likely linked to CYP51A and/or CYP51B gene variants. Phytopathology 2018, 108, 1263-1275. [CrossRef]

32. Muzhinji, N.; Woodhall, J.W.; Truter, M.; van der Waals, J.E. Variation in fungicide sensitivity among Rhizoctonia isolates recovered from potatoes in South Africa. Plant Dis. 2018, 102, 1520-1526. [CrossRef]

33. Shao, W.Y.; Sun, J.T.; Zhang, X.K.; Chen, C.J. Amino acid polymorphism in succinate dehydrogenase subunit C involved in biological fitness of Botrytis cinerea. Mol. Plant Microbe Interact. 2020, 33, 580-589. [CrossRef] [PubMed]

34. He, L.; Li, X.; Gao, Y.; Li, B.; Mu, W.; Liu, F. Characterization and fungicide sensitivity of Colletotrichum spp. from different hosts in Shandong, China. Plant Dis. 2019, 103, 34-43. [CrossRef]

35. Ueyama, I.; Araki, Y.; Kurogochi, S.; Yamaguchi, I. Metabolism of the phenylurea fungicide, pencycuron, in sensitive and tolerant strains of Rhizoctonia solani. J. Pestic. Sci. 1993, 18, 109-117. [CrossRef]

36. Amiri, A.; Heath, S.M.; Peres, N.A. Resistance to fluopyram, fluxapyroxad, and penthiopyrad in Botrytis cinerea from strawberry. Plant Dis. 2014, 98, 532-539. [CrossRef] [PubMed]

37. Weber, R.W.S.; Hahn, M. A rapid and simple method for determining fungicide resistance in Botrytis. J. Plant Dis. Protec. 2011, 118, 17-25. [CrossRef] 